\title{
Mind, Matter, Meaning and Information
}

\author{
Robin Faichney
}

Independent researcher, Dunblane, UK, robin@robinfaichney.org, http://www.robinfaichney.org

\begin{abstract}
This article aims to show how mind, matter and meaning might be united in one theory using certain concepts of information, building on ideas of empathy and intentionality. The concept of intentionality in philosophy of mind ("aboutness"), which is "the ineliminable mark of the mental" according to Brentano, can be viewed as the relationship between model and object, and empathy can be viewed as a form of mental modelling, so that the inclination to attribute mentality can be identified with the inclination to empathise with the relevant entity. Physical information, a concept quite well established within the discipline of physics, is basically a reconceptualization of material form. Daniel Dennett's concept of the intentional stance allows the development of a concept of "intentional information," a broad term that encompasses mental content and semantic information generally, as encoded within physical information/material form.
\end{abstract}

Keywords: Physical Information, Semantic Information, Intentional Information, Mind, Matter, Meaning, Intentionality, Intentional Stance, Mental Model, Cognitive Empathy, Affective Empathy

Acknowledgement: This article is based on a dissertation entitled The Empathic Stance (Faichney 2011) written for the degree of M.Sc. by Research in philosophy, awarded in June 2011 by Edinburgh University, and I am grateful to my supervisor, Dr. Julian Kiverstein, for his help. A very brief version entitled Information and Mind was presented at the Difference That Makes a Difference 2011 workshop at the Open University, Milton Keynes, September 2011, and I am grateful also for feedback received from fellow participants, as well as the comments of two anonymous reviewers and of the guest editor David A. Chapman on the original version of this article.

"It is tempting to suppose that some concept of information [...] could serve eventually to unify mind, matter, and meaning in a single theory." (Dennett \& Haugeland 1987, emphasis in the original)

The aim of this article is to confirm Dennett and Haugeland's supposition. I suggest that information - or to be more precise the relationship between intentional information and material form - is the missing link between mind and matter. ("Intentional" here is a technical term meaning "taking an object" or "aboutness" - see Section 2.) The central contention is that intentional information - a broad but well-defined concept that encompasses mental content, semantic information, and all meaning and significance - is best considered to be encoded in material form, being decoded in use. This can be considered a generalization of Wittgenstein's later theory of linguistic meaning as use (1972).

This theory has a number of implications, but perhaps the most prominent problem that it addresses is what Chalmers (1995) has called "the hard problem" of consciousness. This is the question as to how any physical system, such as a brain, can give rise to subjective experience. I contend that what the attribution of consciousness or mentality (including attribution to self) actually signifies is acknowledgement of the relevant entity as an appropriate object of empathy where that is explained in terms of mental modelling and information. So such attribution depends on the relationship between attributor and attributee, and there can be no objective criterion for it.

Chalmers' own suggested solution to the hard problem involves "a double-aspect view of information" $(1995,200)$ that is in some ways quite similar to mine, but, crucially, lacks an explanation of the relationship between the aspects. Schroeder (2011) also has a superficially similar thesis, but uses what strike me as rather idiosyncratic concepts of information.

Dennett's "intentional stance" is central to my thesis, but I see Dennett's philosophy as lacking an essential ingredient, which is a proper appreciation of empathy. In the first section below, I suggest that empathy has two main forms, cognitive and affective. Cognitive empathy enables the understanding of others' thinking and corresponds to Dennett's concept of the intentional stance, while affective empathy, which Dennett neglects, concerns the sharing of emotion. They are, however, for me, both essentially intentional (in the technical sense of that word), and in Section 2, I suggest that the concept of mental modelling encompasses both and provides a more intuitive and more explanatorily powerful substitute for the concept of intentionality as a defining characteristic of mentality (Brentano 1924). In Section 3 I relate the foregoing concepts to physical information, 
which is a re-conceptualization of material form, and intentional information, which encompasses mental content and meaning in general, and which I argue should be considered encoded in physical information. This is a physicalist account of mind, but one that gives a very prominent place to the essentially pro-social quality of empathy, which should therefore disarm at least some objections to physicalism on broadly humanistic grounds. Though social issues lie beyond the scope of this article (apart from empathy, which I see as the foundation of all social interaction), the reconciliation of humanism with physicalism, and therefore with the natural sciences, must surely be broadly beneficial.

\section{Empathy}

For about twenty five years there have been two main competing theories of folk psychology, but relatively recent research seems to indicate that, properly understood, they are both valid, and indeed complementary. They correspond to two forms of empathy.

A substantial proportion of social interaction consists of, or relies upon, the attempted prediction by one person of another's future actions, or the explanation of past actions. It is, perhaps, a necessary component of your belief that you "know someone" that you think you can successfully predict how they would act in some situations. And, of course, there are some situations in which we think all normal people would behave similarly: finding themselves in danger, for instance, they would seek to escape from or mitigate that danger. So people generally have some understanding of each other, and this understanding has commonly been called "folk psychology."

The main alternative theories of folk psychology are the "theory-theory" and the "simulation theory." The former takes folk psychology to be a quasi-scientific activity, involving entities such as beliefs and desires and hypotheses about law-like regularities governing their interactions. The alternative theory is that we "simulate" other people's minds, in order to predict and explain their behaviour, imaginatively putting ourselves in their situation. Both of these strategies can be classified as forms of empathy. Hybrids of theory-theory and simulation theory, suggesting that we use both strategies, have also been suggested. The first subsection below is concerned with theorytheory, the second with simulation theory, and the third with hybrids and empathy.

\subsection{Theory-Theory}

The term "theory of mind" was first used in psychology by Premack and Woodruff (1978). Their work with a chimpanzee named Sarah seemed to demonstrate that she attributed desires to a human pictured in certain dilemmas, which she "solved" for him by selecting pictures depicting the appropriate action on his part. The authors suggested that the system of inferences implied by Sarah's choices "...is properly viewed as a theory" (Ibid., 515). Premack and Woodruff's theory accordingly became known as the "theory-theory".

Baron-Cohen et al. (1985) later designed an experiment to test young children, which has since been repeated with various modifications, being generally referred to as "the Sally-Anne test," after the characters in the scenario, or "the false-belief test."

Sally and Anne are two dolls. Sally has a basket, and Anne, a box. The child is shown Sally putting a marble in her basket and then leaving the room. While Sally is out, Anne takes the marble from Sally's basket and puts it in her own box. Sally then returns, and the child is asked where Sally will look for her marble. The correct answer is "in the basket," but very young children appear to have no concept of false belief, and say "in the box," presumably because that is where they know the marble is. Older children, however, from around four years of age, generally "pass" the test (Baron-Cohen et al. 1985): they correctly attribute a false belief to Sally, having, according to the theory-theory, become able to recognise that other people have minds of their own. This is the concept of the child as "little scientist." (Gopnik and Meltzoff 1997)

\subsection{Simulation Theory}

A rival explanation emerged in the mid-eighties. Heal (1986) and Gordon (1986) independently suggested that, rather than theorizing about Sally's mental state, the child would imagine herself in Sally's position, or "put herself in Sally's shoes," enabling her to simulate Sally's thinking and behaviour.

The basic idea is that if the resources our own brain uses to guide our own behaviour can be modified to work as representations of other people, then we have no need to store general information about what makes people tick: we just do the ticking for them (Gordon 2009). 
Gallese and Goldman (1998) use findings concerning "mirror neurons" in macaque monkeys to support an argument for simulation theory. Mirror neurons are so named because they fire both when the animal makes a certain action and when it observes another performing the same action. Individual mirror neurons have not been found in humans (there are problems with recording single cell activation in humans), but studies using various neuroimaging techniques have revealed "mirror systems" that include the areas in which mirror neurons have been found in monkeys, but also others including the somatosensory cortex, which, it has been suggested, allow people to know what it feels like to perform the observed action (Gazzola and Keysers 2009).

\subsection{Empathy and Hybrid Theories}

Theodor Lipps, writing in German on aesthetic appreciation (1903), coined the term Einfühlung, literally "feeling-into", which came to be translated as "empathy". (Wispé 1990) He believed that it is through empathy that we come to know others: we do not perceive such emotions as pride, shame, anger, sorrow, and joy in others directly, but experience them vicariously. We "feel for" the other person.

The psychologist G.W. Allport disagreed. He argued that knowledge about others must be something more than empathy. A "proud" gesture or a "joyous" laugh, for example, describes those qualities in another sentient being, and so first there must be a realization of the consciousness of the other. There can be no proud or joyous stones. (Allport 1937)

However, while logic might seem to dictate that belief in the consciousness of another must precede any belief regarding the content of that consciousness, Sytsma and Machery (2010) provide experimental evidence that, for non-philosophers, empathy comes first and rationalization concerning the status of the empathic object later. In psychological terms, there is no reason why sharing of emotion should not come first and rationalization later, and this view would seem to be supported by the implication of the neurological evidence (see below) that empathic tendencies are innate. Allport has it back-to-front: he thinks that he must know that the other is conscious before he can empathize with them, but in fact he only wishes to attribute consciousness because he finds himself tending to empathize.

Despite its relatively recent coinage, there are now many different definitions of empathy. Shamay-Tsoori reviews the literature and finds a number of different concepts, dividing them into two categories: "The critical difference between cognitive empathy and affective or emotional empathy is that the former involves cognitive understanding of the other person's point of view whereas the latter also includes sharing of those feelings..." (Shamay-Tsoori 2009, 215). Following brief descriptions of theory-theory and simulation theory, in connection with which she mentions mirror neurons, Shamay-Tsoori goes on to suggest that the former:

"...views empathy as a thoroughly "detached" theoretical analysis that involves areas of the cortex that are usually activated during mental state attribution, whereas simulation depicts empathy as incorporating an attempt to replicate the other's affective mental state via neural networks related to emotion processing. ... [I]t may be suggested that cognitive empathy involves more [theory] processing, whereas affective empathy involves more simulation processing." (Ibid., 216)

Shamay-Tsoori implies that mirror systems reflect emotions as well as actions and Watson and Greenberg make this explicit: "Regions in the brain associated with feeling a specific emotion are activated by seeing that emotion in another or witnessing the other in a situation that might elicit the emotion" (Watson and Greenberg 2009, 126). More specifically, Pfeifer and Dapretto report that in children asked to imitate or just observe various emotional facial expressions there are "significant correlations between activity in mirror neuron and limbic regions and each of the first three subscales of the Interpersonal Reactivity Index" (Pfeifer and Dapretto 2009, 188). (The Interpersonal Reactivity Index is a widely used test of empathy of which these subscales measure the more affective components.)

Affective empathy plays rather an important role here, and there is one definition of it in the literature that fully accords with my own view:

There is empathy if: (i) one is in an affective state; (ii) this state is isomorphic to another person's affective state; (iii) this state is elicited by the observation or imagination of another person's affective state; (iv) one knows that the other person is the source of one's own affective state. (de Vignemont and Singer 2006, 435)

Categorization of empathy into cognitive and affective varieties, and the close association of these with theory-theory and simulation theory respectively, are quite solid findings. As elaborated 
in my M.Sc. dissertation (Faichney 2011), we attribute sentience, the capacity to perceive and to experience pleasure and suffering, to the entities towards which we experience a tendency to affectively empathize. Similarly, we attribute cognition, or the capacity for thought, to the entities towards which we experience a tendency to cognitively empathize. (We very often attribute sentience and cognition on the basis of membership of a species, or other grouping, rather than empathy with the particular individual concerned, but that seems clearly inferable from the experience of the tendency to empathise on a one-to-one basis.) Of course, in the case of other people, both tendencies are generally present, and the principal feature that they share, intentionality, is the subject of the next section.

\section{Intentionality}

In philosophy of mind "intentional" is a technical term that means not "deliberate," but "taking an object," rather like grammatical transitivity. ${ }^{1}$ So intentions are intentional, in this sense, being always to do something, but beliefs, desires, hopes, fears, loves, hates, etc. are all object-directed too, and are therefore intentional in this technical sense. According to Brentano, in fact, all mental phenomena, and no physical phenomena, are intentional. Re-introducing the concept from medieval philosophy, he called it "the ineliminable mark of the mental" (Brentano 1924). I proceed on the assumption that intentionality is such a distinguishing characteristic, but whether it is eliminable is another matter, to which I will return.

Daniel Dennett's magnum opus is Consciousness Explained (1991a), but in the appendix for philosophers (the book is aimed at a general readership) he mentions "the other half of my theory of mind" (1991a, 457), the theory of mental content that he calls "the intentional stance". That is part of an array that contains two other stances, physical and design, but the design stance is not directly relevant here and I will confine myself to a very brief description of the physical stance before going on to deal with the intentional one.

The physical stance is what we use when we think of and deal with a physical object qua physical object, such as a rock that might or might not be kicked, depending on its likely mass and the type of footwear being worn at the time: the outcome depends entirely upon physical factors (assuming the inclination to kick a rock in the first place).

The intentional stance operates at a higher level of abstraction. "With their wings" is an answer to the question as to how winged creatures fly (if not a very informative one), but why do they do so? For many reasons, probably, but let's take as an example what's certainly a common one: to find food. In order to understand that we view the bird as desiring food and believing that it can be found some distance away - far enough to make flying a better way of getting there - than walking, hopping, swimming, etc. Belief and desire are intentional concepts; the archetypal ones in discussions of intentionality, though there are many others. They are about something: desire for food, belief that it might be found somewhere. We might argue about the details of avian psychology, but it would be extremely difficult while doing so, perhaps even impossible, to dispense altogether with intentionality. Dennett explains it thus:

"Here is how it works: first you decide to treat the object whose behaviour is to be predicted as a rational agent; then you figure out what beliefs that agent ought to have, given its place in the world and its purpose. Then you figure out what desires it ought to have, on the same considerations, and finally you predict that this rational agent will act to further its goals in the light of its beliefs." (Dennett 1987, 17)

I assume that a bird does not consciously reason, is not rational in that sense, but its innate behavioural traits are rational in the appropriate context given its environmental niche and the evolutionary imperative to survive and reproduce. This use of the evolutionary perspective might seem surprising, but, for Dennett, a bird is not conscious. Dennett equates sentience with mere "mechanical sensitivity" (1997), and so to consider what an individual might have in mind at a particular time is inappropriate. Indeed, Dennett's bird, unlike mine, has no mind. I argue that birds and other animals have minds because (to anticipate) they are capable of suffering and enjoyment, and capable of appropriate action in response to pleasant and unpleasant stimuli.

What is probably most controversial here is that for Dennett (and here I agree with him) this is entirely a matter of interpretation. No system is intrinsically intentional: "...a particular thing is an intentional system only in relation to the strategies of someone who is trying to explain and predict its behaviour" (Dennett 1971, 87). This is undoubtedly a difficult point. We seem intrinsically inclined to regard ourselves as intrinsically intentional systems. But it is an important feature of Den-

\footnotetext{
${ }^{1}$ Saraceni (2008) discusses intentionality in relation to grammatical transitivity.
} 
nett's philosophy that in principle anything and everything can be explained using the physical stance, while to adopt either of the higher level stances (intentional or design) is merely a matter of convenience - even though it can, in practice, make the difference between impossibility and ease (of prediction or explanation). Contra Brentano, intentionality is, in principle, eliminable. But I have more to say about this below.

As already mentioned, I follow Brentano in seeing intentionality as a feature that unambiguously distinguishes mental from physical phenomena. Why should this be, what is it that links mentality and intentionality so closely? My answer is that mentality necessarily implies representation or modelling (in the sense of mathematical or computer modelling), and intentionality is simply the relationship between model and object: all "aboutness" is fundamentally of this nature. So to view an entity as a modeller is to adopt the intentional stance towards it. But modelling is a more intuitive concept than intentionality, and so to view minds as necessarily incorporating models leads to better explanations than does insisting on intentionality as the distinguishing characteristic of mental phenomena.

While all minds are modellers and necessarily intentional, not all that is intentional is a modeller or a mind. Take this article as an example: it, like all communications, is composed of intentional information. That is discussed in Section 3.2, but, to anticipate, I will say here that intentional information always constitutes part, at least, of a model.

Minds do not just passively represent their surroundings, though: they act to change things, to make them more as they would like them to be. A minimal mind (that of the simplest creature to which we feel inclined to attribute sentience) will use at least two models (or sets of models), one of present reality (beliefs) and another of a desired state or states (desires), and will tend to act to reconcile these by changing reality (as perceived/believed) to bring it into closer alignment with the desired state. Purpose and use are therefore implied by mentality. But we should not lose sight of the fact that, modelling/intentionality being essential to it, mentality is a matter of interpretation, on this account. It is the belief/desire modelling arrangement that gives meaning to "purpose" and "use" - and, indeed, "meaning," see Section 3.2 - otherwise there is nothing but merely mechanical activity, which is another way of saying that we are reduced to using the physical stance.

It is largely the innate nature of our tendency to empathise with each other that encourages us to view ourselves as intrinsically intentional. However, another important factor is our awareness of our selves as conscious beings. This can be understood in terms of second order modelling, which encompasses both the modelling of other modellers and self-modelling (Faichney 2011). The capacity to model a self - any self - is more fundamental than the distinction between my self and other selves. It is not possible to do justice to this aspect of the theory here, but the utility of the intentional stance is such that the theoretical eliminability of intentionality is, for all social and practical purposes, irrelevant.

As mentioned at the beginning of this section, Dennett's "great work" is Consciousness Explained (1991a), but while this has been fairly influential regarding typical adult human consciousness, even some of those who are quite well-disposed towards it have questioned whether Dennett is right to restrict consciousness to thinkers, or, as Clark puts it, whether "experiences need a thick subject" (Clark 2002, 197), ${ }^{2}$ and Blackmore (2003) claims that her experience of meditative consciousness cannot be accounted for by Dennett's theory. It would be beyond the scope of this article to address this issue properly, but I attempt to do so in my M.Sc. dissertation (Faichney 2011), following Robbins and Jack (2006). Robbins and Jack suggest an addition to Dennett's array of stances that they call "the phenomenal stance." This extends experience to those entities that would generally be considered merely sentient (including, in my view, unthinking meditators), rather than restricting it to the subjects of full-blown Dennettian consciousness. I differ from Robbins and Jack on one point, though, in that the stance that I would add to Dennett's array is, unlike the phenomenal stance, intentional (Faichney 2011), so that there would be two intentional stances. It is not possible here to go into my reasons for taking that position.

Robbins and Jack go to some lengths to identify Dennett's intentional stance with cognitive empathy, and their phenomenal stance with affective empathy, implying, persuasively in my view, that Dennett neglects the latter. On this account it is due to the affective empathy that we tend to experience towards them that we feel inclined to attribute experience, primarily of suffering and pleasure but also of perception generally, to creatures much simpler than ourselves, as well as each other. ${ }^{3}$

\footnotetext{
2 "Thick," there, derives from IT jargon, and means roughly the opposite of the word's common colloquial (UK) use: a thick subject is a complex, sophisticated one.

${ }^{3}$ See also Sytsma and Machery (2010), who suggest that, "For the folk," unlike philosophers, "subjective experience is tightly linked to valence" $(2010,300)$, "valence" in this context meaning positive or negative hedonic value: pleasure and pain.
} 
In these respects my additional stance is identical to that of Robbins and Jack. As mentioned, however, mine, unlike theirs, is intentional. Obviously, in an arrangement where there are two intentional stances, one of which is generally referred to as "the intentional stance," there is great scope for confusion, but the substitution of modelling for intentionality in the context of understanding mentality reunites these stances, as, when we attempt to understand other people, we model both their emotionality and their cognition.

\section{Physical and Intentional Information}

There are, notoriously, many concepts of information, and I do not claim that those described here are superior to any others in general terms. For Dretske $(1981 ; 2000)$, for instance, information is akin to meaning, except that whereas a meaningful statement might or might not be true, information, to be informative, must be true. While this is a common and, indeed, useful concept, my use of the term is better suited to the sort of theoretical analysis that I attempt here. The more important of the many uses of "information" are surveyed by Floridi (2004). The first of the two concepts utilized here, physical information, comes from physics. The second, intentional information, is my own coinage, and is an intuitive generalization of the common concept of semantic ("meaningful") information, which need not be true. The relationship between physical and intentional information is central to the current thesis. The application of en/decoding and related concepts to natural phenomena, in the first subsection below, is perhaps to some degree original, but rather obvious given the concept of physical information. My main contribution is in the second subsection: the concept of intentional information and its relationship with physical information.

\subsection{Physical Information}

At a conference in Dublin in July 2004, the physicist Stephen Hawking admitted he'd made a mistake (Preskill 2004). He had bet another physicist, John Preskill, that black holes destroy information, but Hawking changed his mind, conceded publicly at the conference, and presented Preskill with his prize: an encyclopaedia (to represent enduring information). Hawking had come to believe that information is preserved inside black holes, and eventually escapes them. A black hole, he now agreed, behaves like a computer: input information is transformed into output information in a systematic way that could, in principle, be deduced by comparing input and output. Anything that falls into a black hole is considered input information. The output is in the form of "Hawking radiation," an earlier postulate of his. If it were possible to monitor all that falls into a black hole, and all of the Hawking radiation that emerges from it, it would be possible in principle to discover all that goes on within it.

According to physicist Roy Frieden (1998), the laws of physics are generated by the attempt to minimize the difference between an entity or system's own physical information, and the information that physicists can obtain about it. That particular suggestion remains controversial, but the concept of physical information is now very well established (for a useful overview of information in physics see Vedral (2012)). It developed from the concept of information in communication theory (Shannon 1948), and like that has no semantic aspect or component. Physical information is basically material form, which, using mathematical techniques deriving from communication theory, can be quantified. It might be thought of as shape, but the concept is actually much more general, encompassing all physical qualities. So any physical entity can be considered to embody its own description, if its form (in that general sense) is treated as physical information.

To experiment upon a physical object is to seek to extract its physical information, but there are two complicating factors that need to be considered. Firstly, once obtained, information about the object is not physical information, despite its referent being entirely physical. Secondly, the information obtained directly from the experiment actually concerns not the object alone, but the interaction between that and the experimental apparatus, whatever that might be. To obtain information about the object, further processing is required.

This brings us to an important principle: every physical thing encodes the outcomes of all of its potential interactions (which is in fact implied in saying that it embodies its own description). This is most easily envisaged using a simple case, where there are just two things, such as two asteroids on a collision path: each of them can be considered to encode the aftermath of the collision, where the other is the decoding key.

Now, what is encoded anywhere is necessarily (but loosely speaking) "a matter of opinion": it depends on the decoding key, and different keys elicit different messages. Of course, in the case of human communications, a particular message is intended to be conveyed, and that is the criterion of correct decoding. However, the concepts of en- and decoding are generalized here, being ap- 
plied to natural phenomena, where that criterion does not apply. It is a trivial consequence of considering material form as information that a state of affairs at one time can be considered encoded in a related state of affairs at another time. In the case of the asteroids, either one can be considered to encode the outcome of the collision, simply by designating the other as the key. So every physical entity can be considered to encode the outcomes of all of its potential interactions. The only constraint is context (I discuss the relationship between key and context below). Where genes are considered to be, not DNA sequences, but encoded in DNA sequences (Dennett 1995), the cellular context is what limits the outcome of DNA molecule interactions to (generally) biologically valid configurations.

Turning again to our asteroids, in reality there will always be influences, such as gravitation, also from other objects. Ultimately, every physical thing is continuously interacting with the rest of the universe. However, some interactions are more significant than others. The distinctions between decoding key, context and background are entirely relative: the most important interacting entity, where one can be identified, will usually be considered the key, while the less important but nonnegligible entities constitute the context, and those that can safely be ignored are relegated to the background. But in some cases we need to allow for many influences, and there we can consider the context to be the decoding key.

\subsection{Intentional Information}

It was stated above that information obtained by experimenting upon a physical thing is not itself physical. This is what I call intentional information: information that is about something, whether the referent is real or not, whether or not the information is true.

The concept of intentional information has two major virtues: it is a general term, encompassing mental content and all meaning, reference, significance, etc.; and the relationship between it and physical information - and therefore, ultimately, between mind and meaning on one hand and matter on the other - can be stated quite simply: intentional information is always encoded in physical information. The outcome of an interaction between two asteroids is similarly encoded, though, so what distinguishes intentional information? Using the relationship between intentionality and modelling suggested in Section 2, we can say that intentional information is that which plays a part in a model.

But the crucial point is this: the encoding of intentional information in physical information, and its consequently context-dependent nature, accurately reflect the characteristics of subjectivity: uncertainty, relativity to point of view, and so on. In particular, the context-dependent nature of en/decoding reflects the dependency of intentionality and modelling on interpretation (Section 2).

What is the ontological status of intentional information?

I hope that it is safe to assume that the reality of physical information or material form is uncontroversial. Dennett argues in Real Patterns (1991b), roughly speaking, that a pattern is real if and only if it can be compressed without loss (following Chaitin (1975)). Now, this sort of information, which might broadly be characterised as "mathematical," is neither physical nor intentional, but it shares features with both of these. Like intentional information, it is always encoded in physical information, but like the latter it does not refer to or mean anything ${ }^{4}$-it could be viewed as "pure pattern." So what I take from Real Patterns is that patterns encoded in physical information can be considered real. ${ }^{5}$

Intentionality, though, is a matter of interpretation (Section 2). That necessarily applies to intentional information as such, but not to the patterns concerned. This becomes clearer when an appropriate example is chosen: the patterns of paint on a canvas are perfectly real, while the degree of realism of the scene represented is a matter of opinion. Like the paint, the neural and other patterns that serve as the vehicles for intentional information are actually physical, however complex, distributed or otherwise difficult to discern. Ultimately, there is only physical information, because intentional information is actually an aspect of the use of physical information, ${ }^{6}$ rather than any kind of entity in itself, and so claims regarding it are therefore not ontological in nature. The concept of intentional information was inspired by the later position of Wittgenstein on meaning (1972), of which this as a generalization. Intentional information is dependent on use and therefore on mind, and has no independent existence, though the patterns that are used are entirely real.

Due to our life-long, habitual, indeed utterly unavoidable use of it, intentional information for us appears quite explicit. However, in "the objective, materialistic, third-person world of the physical

\footnotetext{
${ }^{4}$ To view physical information as referring to the object that instantiates it is a mistake, because it is the form of that object, an integral aspect of it.

"I believe this position also to be consistent with Ross's "rainforest realism" (2000).

${ }^{6}$ Recall Chalmers' dual aspect theory of information mentioned in the preamble.
} 
sciences" (Dennett 1987, 5), which implies the physical stance, intentional information simply does not exist.

On this account, consciousness can be viewed as a stream of intentional information, the experience of which is what it is like to be a user of information, the minimal example of which is the sentient modeller described in Section 2. When a beam of light entering your eye carries information about an apple, that information is encoded. The encoding takes place when the light encounters the surface of the apple and is filtered by the structures it finds there as it is reflected, so the balance of the mixture of wavelengths within it is changed. The decoding takes place within the eye, the optic nerve and the brain, as that particular mixture of wavelengths is interpreted to be the colour of the apple, and other features are similarly deduced. Only the light's own physical information enters the eye, but the chain of causation means that the light can be processed to yield information about the apple. The physical information of the light is the carrier, the brain etc. is the decoding mechanism, and the apple's colour, etc. is the coded message. Without the intentional stance, there is just the physical information of the brain's structure and activity (however complex), but with the intentional stance, some of that physical information can be taken to encode intentional information about things outside the brain. Intentionality uses physical information while obscuring the details of that use. In particular, it involves viewing causal chains as relatively simple, and necessary rather than contingent, resulting in the assumption of some kind of relationship between subject and object over and above that of the actual complex and often fragile causal chain. The temptation to believe in such a "special relationship" is very largely due to the amazingly reliable and successful nature of our everyday interactions with the things and people around us. Intuition is misled by the automatic and systematic concealment of complexity.

\section{Summary and Conclusions}

The phrase "theory of mind" has two different meanings, depending on whether the context is philosophy or psychology. What I have presented here is a philosophical theory of mind that involves the psychological concept (Section 1.1). The connection is that our overarching theories about persons (philosophy) should be informed by the ways in which people actually relate to each other (psychology).

I have taken the philosophy of Daniel Dennett as a starting point, but, following Robbins and Jack (2006), modified it so that the concepts of mind and consciousness can legitimately be applied to creatures much simpler than ourselves. This was accomplished by identifying Dennett's concept of the intentional stance with cognitive empathy, then arguing that he neglects affective empathy. With an intentional stance that encompasses affective as well as cognitive empathy, we have the basis of viewing simple creatures as capable of experiences, primarily pleasure and pain.

The motivation for empathising with simple creatures is thus emotional. We affectively empathise also with other humans, of course, but with them we can also empathise cognitively, thus seeking to understand their thoughts as well as their emotions. One of the central contentions here is that the main psycho-social significance of the attribution of consciousness or mindedness is empathy: when I decide to treat an entity as conscious, what that means is that I regard it as an appropriate object of empathy - and thus not just an object but another subject.

I differ from Robbins and Jack in taking a strongly intentionalist approach, so that what they call "the phenomenal stance" is for me as intentional as Dennett's "intentional stance". I have not presented an argument for this point here. Nevertheless, it is significant due to Dennett's analysis of intentionality as a matter of interpretation. While my concepts of consciousness and mindedness are much more inclusive than Dennett's, they are equally interpretative. This is highly counterintuitive, but I believe the evidence is such that conflicting intuitions must ultimately be abandoned. There can be no objective criterion for consciousness or mindedness, because attribution depends entirely upon the relationship between attributor and attributee - even when one person plays both roles (another point upon which I have not been able to elaborate here).

"Reduction" of the attribution of consciousness and mindedness to the tendency to empathise constitutes one side of the philosophical theory of mind presented here. The other side concerns information. Physical information, a concept well established in physics, is a reconceptualization of material form. Intentional information, my own concept, corresponds approximately to the common concept of semantic information, but is very broad, encompassing mental content and all meaning and significance. Intentional information is always encoded within physical information, or material form, being decoded (and in the case of human communications encoded) in use. This view was inspired by Wittgenstein's later concept of linguistic meaning as use, and can be seen as a generalization of it. It explains the relationship between mind and matter, thus solving Chalmers' "hard problem of consciousness" (1995). 
A criticism sometimes levelled at such physicalist theories of mind, and which very often seems to lie behind such criticism even when not explicit, is that of negative normative and/or ethical and/or political implications. The assumption seems to be that to "explain away" mind or consciousness in "merely" physical terms is to discourage empathy. (Similarly, non-libertarian theories of free will are often, I believe, assumed to discourage aspiration.) Physicalist philosophers would tend to argue against such criticisms, saying they are based on misunderstandings, or perhaps in some cases would claim that they are interested only in discovering the truth, that pursuit being ultimately a beneficial one. In this case, however, empathy is "front and centre", and whatever criticisms might be levelled at the theory described above, the discouragement of empathy surely is not one of them. Indeed, I believe that a proper appreciation of the significance of empathy would not merely counter any otherwise negative effects of physicalism, but encourage a conscious effort constantly to improve one's understanding of other people.

\section{References}

Allport, Gordon. 1937. Personality: A Psychological Interpretation. New York: Henry Holt.

Baron-Cohen, Simon, Alan Leslie, and Uta Frith. 1985. Does the Autistic Child Have a "Theory of Mind"? Cognition 21(1): 37-46.

Blackmore, Susan. 2003. Consciousness in Meme Machines. Journal of Consciousness Studies 10 (4-5): 19-30.

Brentano, Franz. 1924. Psychologie vom Empirischen Standpunkt (Bd. 1) [Psychology from an Empirical Perspective]. Leipzig: Meiner. Original work published in 1874

Chaitin, Gregory. 1975. Randomness and Mathematical Proof. Scientific American 232, 47-52.

Chalmers, David. 1995. Facing Up to the Problem of Consciousness. Journal of Consciousness Studies 2(3): 200-219.

Clark, Andy. 2002. That Special Something: Dennett on the Making of Minds and Selves. In Daniel Dennett, edited by Andrew Brook and Don Ross, 187-205. Cambridge: Cambridge University Press.

Davies, Martin, and Tony Stone. 2001. Mental Simulation, Tacit Theory, and the Threat of Collapse. Philosophical Topics 29(1\&2): 127-73.

de Vignemont, Frederique, and Tania Singer. 2006. The Empathic Brain: How, When and Why? Trends in Cognitive Sciences 10(10): 435-441.

Dennett, Daniel. 1971. Intentional Systems. The Journal of Philosophy 68(4): 87-106.

Dennett, Daniel. 1987. The Intentional Stance. Cambridge, Massachusetts: MIT Press.

Dennett, Daniel. 1991a. Consciousness Explained. London: Allen Lane.

Dennett, Daniel. 1991b. Real Patterns. Journal of Philosophy 87, 27-51.

Dennett, Daniel. 1995. Darwin's Dangerous Idea: Evolution and the Meanings of Life. New York: Simon \& Schuster.

Dennett, Daniel. 1997. Kinds of Minds: Toward an Understanding of Consciousness. New York: Basic Books.

Dennett, Daniel. and John Haugeland. (1987). Intentionality. In The Oxford Companion to the Mind, edited by Richard L. Gregory, 383-386. Oxford: Oxford University Press.

Dretske, Fred. 1981. Knowledge and the Flow of Information. Cambridge: MIT Press.

Dretske, Fred. 2000. Perception, Knowledge, and Belief: Selected Essays. Cambridge: Cambridge University Press.

Faichney, Robin. 2011. The Empathic Stance. M.Sc. Dissertation, Edinburgh: University of Edinburgh. Accessed November 22, 2011. http://www.robinfaichney.org/pdf/MScDissertation.pdf

Floridi, Luciano. 2004. The Blackwell Guide to the Philosophy of Computing and Information. Oxford: Blackwell.

Frieden, B. Roy. 1998. Physics from Fisher Information. Cambridge: Cambridge University Press.

Gallese, Vittorio, and Alvin Goldman. 1998. Mirror Neurons and the Simulation Theory of Mind-Reading. Trends in Cognitive Sciences 2(12): 493-501.

Gazzola, Valeria, and Christian Keysers. 2009. The Observation and Execution of Actions Share Motor and Somatosensory Voxels in all Tested Subjects: Single-Subject Analyses of Unsmoothed fMRI Data. Cerebral Cortex 19(6): 1239-1255.

Gopnik, Alison, and Andrew Meltzoff. 1997. Words, Thoughts, and Theories. Cambridge: MIT Press.

Gordon, Robert. 1986. Folk Psychology as Simulation. Mind and Language 1(2): 158-171.

Gordon, Robert. 2009. Folk Psychology as Mental Simulation. Stanford Encyclopedia of Philosophy. Accessed December 8 , 2009. http://plato.stanford.edu/entries/folkpsych-simulation/

Heal, Jane. 1986. Replication and Functionalism. In Language, Mind and Logic, edited by Jeremy Butterfield, 135-150. Cambridge: Cambridge University Press.

Lipps, Theodor. 1903. Einfühlung, Inner Nachahmung, und Organ-Empfindungen. Archiv für die gesamte Psychologie 2: 185-204

Pfeifer, Jennifer, and Mirella Dapretto. 2009. "Mirror, Mirror, in my Mind": Empathy, Interpersonal Competence, and the Mirror Neuron System. In The Social Neuroscience of Empathy, edited by Jean Decety and William Ickes, 183-198. Cambridge, Massachusetts: MIT Press.

Premack, David, and Guy Woodruff. 1978. Does the Chimpanzee have a Theory of Mind? Behavioral and Brain Sciences 1(4): $515-526$.

Preskill, John. 2004. On Hawking's Concession. Accessed November 18, 2011. http://www.theory.caltech.edu/ preskill/jp 24jul04.html

Robbins, Philip, and Anthony Jack. 2006. The Phenomenal Stance. Philosophical Studies 127(1): 59-85.

Ross, Don. 2000. Rainforest Realism: A Dennettian Theory of Existence. In Dennett's Philosophy: a Comprehensive Assessment, edited by Don Ross, Anthony Brook, and David Thompson, Cambridge: MIT Press.

Saraceni, Mario. 2008. Meaningful Form: Transitivity and Intentionality. ELT Journal 62(2): 164-172.

Shamay-Tsoori, Simone. 2009. Empathic Processing: Its Cognitive and Affective Dimensions and Neuroanatomical Basis. In The Social Neuroscience of Empathy, edited by Jean Decety and William Ickes, 215-232. Cambridge: MIT Press.

Shannon, Claude. 1948. A Mathematical Theory of Communication. Bell System Technical Journal, 27: 379-423, 623-656. 
Schroeder, Marcin. 2011. Concept of Information as a Bridge between Mind and Brain. Information 2: 478-509.

Sytsma, Justin, and Edouard Machery. 2010. Two Conceptions of Subjective Experience. Philosophical Studies 151(2):

299-327.

Vedral, Vlatko. 2012. Information and Physics. Information, 3: 219-223.

Watson, Jeanne, and Leslie Greenberg. 2009. Empathic Resonance: A Neuroscience Perspective. In The Social Neuroscience of Empathy, edited by Jean Decety and William Ickes, 125-138. Cambridge: MIT Press.

Wispé, Lauren. 1990. History of the Concept of Empathy. In Empathy and its Development, edited by Nancy Eisenberg and Janet Strayer, 17-37. Cambridge: Cambridge University Press.

Wittgenstein, Ludwig. 1972. Philosophical Investigations. Translated by Gertrude Anscombe. Oxford: Blackwell, First published in 1953.

\section{About the Author}

Robin Faichney

graduated B.A. (Hons.) in philosophy and psychology from the University of Stirling, 1981, and M.Sc. in information technology, CNAA, 1986. Since 1986 he has worked in information technology but, having retained a keen interest in philosophy of mind since his first degree studies, he took advantage of a change in personal circumstances to return to formal study and graduated M.Sc. by Research in philosophy from the University of Edinburgh in 2011. He currently pursues independent research. 